\title{
Chemotherapy induced pseudo-cirrhosis in a breast cancer patient with liver metastasis: A case report and review of the literature
}

Ziv Lahav, Mohamed Mowaswes, Bashar Fteiha, Ilia Davarashvili, Saleh Daher, Abu Rmeileh Ayman, Tawfik Khoury* Department of Internal Medicine, Hebrew University-Hadassah Medical Center, Jerusalem, Israel

Received: November 10, 2015

DOI: $10.5430 /$ crim.v3n1p51
Accepted: January 15, $2016 \quad$ Online Published: January 17, 2016

URL: http://dx.doi.org/10.5430/crim.v3n1p51

\begin{abstract}
We report a case of 55-year-old female patient with past medical history of left breast invasive ductal carcinoma in situ who was admitted to the hospital due to ascites and peripheral edema. Following an extensive investigations, the patient was diagnosed with chemotherapy induced pseudo-cirrhosis which developed after chemotherapeutic treatment with cyclophosphamide/adriamycin followed by cisplatin and gemfibrozil for recurrent breast cancer with, lungs, liver and bone metastasis which progressed to clinical cirrhosis. The patient was managed with aggressive diuresis and albumin infusions, however without resolution of both the clinical symptoms of cirrhosis and the pseudo-cirrhotic appearance of the liver. This case demonstrates that the development of chemotherapy induced pseudo-cirrhosis can rapidly progress to true clinical cirrhosis with its life-threatening complications. Thus, clinicians and radiologist should be well aware of this entity as early recognition and management can lead to a near complete recovery of liver function and much improved quality of life.
\end{abstract}

Key Words: Breast cancer, Liver metastasis, Pseudo-cirrhosis, Chemotherapy

\section{INTRODUCTION}

Pseudo-cirrhosis is the development of hepatic nodules after the initiation of chemotherapy for the treatment of metastatic liver as well as for primary liver malignancies. ${ }^{[1,2]}$ In particular, pseudo-cirrhosis was reported in liver metastasis from breast cancer. ${ }^{[1-5]}$ However several other malignancies with liver metastasis have been reported to be associated with pseudo-cirrhosis, including metastatic colon and pancreatic cancer. ${ }^{[6]}$ Usually, pseudo-cirrhosis does not show the clinical features of true cirrhosis associated with decreased liver synthetic function. ${ }^{[7]}$ The suggested mechanism of pseudo-cirrhosis is attributed to the tumor tissue retraction and scarring secondary to chemotherapeutic treatment. The process of hepatic tissue retraction typically occurs near the metastatic lesion after the initiation of chemotherapy and in most instances can develop rapidly over 1-3 months. ${ }^{[3]}$ It is extremely important that these changes are not be mistaken for the development or progression of liver metastasis. Physicians should be aware that hepatic capsular retraction is common in patients with hepatic metastasis from breast cancer, and cases have been reported with extreme capsular retraction. ${ }^{[8]}$ Herein, we report a case of chemotherapy induced pseudo-cirrhosis in patient with metastatic breast cancer who progressed to true clinical cirrhosis without resolution following discontinuation of the chemotherapeutic treatment.

\footnotetext{
*Correspondence: Tawfik Khoury, MD; Email: tawfikkhoury1@ hotmail.com; Address: Department of Internal Medicine, Hebrew UniversityHadassah Medical Center, Jerusalem, Israel.
} 


\section{CASE PRESentation}

We report a case of a 57-year-old female patient, who was diagnosed in 2006 with invasive ductal carcinoma in situ of her left breast. Following a lumpectomy, the patient received chemotherapy after which she was treated with tamoxifen for a year and a half, and later on hormonal treatment with letrozole. In October 2014, on account of elevated cancer antigen 19-9 (CA19-9) level the patient underwent breast magnetic resonance imaging (MRI) which did not demonstrate any recurrence of disease in the breasts, an ultrasound demonstrated right axillar lymphadenopathy, from which a biopsy proved a metastatic breast cancer. Further investigations included, an abdominal MRI and positron emission tomography (PET-CT) from January 2015 which demonstrated findings consistent with liver hemangiomas and evidence of lung and liver metastasis without signs of cirrhosis. The patient was treated with tamoxifen for six weeks followed by chemotherapy with cyclophosphamide and adriamycin every two weeks and was schedule to receive four courses, however, following the first course the patient developed neutropenia, on account of which the chemotherapy plan was switched in June 2015 to gemfibrozil/cisplatin, of which she received six courses. After half a year, the patient started to complain of bilateral leg edema, abdominal swelling and appearance of jaundice. A repeated PET-CT demonstrated close to complete resolution of the pulmonary metastasis, shrinking of the right axillary lymph nodes, and interestingly, the liver this time had a lobulated contour with new hypodense regions, the liver metastasis demonstrated in the previous exam were mostly resolved. The parenchymal changes are consistent with chemotherapy induced pseudocirrhosis. Despite the aforementioned findings, the patient has received an additional course of 5FU and an additional elevation in liver enzymes was seen, the patient was then transferred to our institution for further evaluation. Upon admission, the patient was afebrile 36.6 degree celsius, BP $95 / 64 \mathrm{mmHg}$, pulse rate of 104 beats per minute, room air saturation of $94 \%$, without evidence of encephalopathy, her physical examination was unremarkable except for deep jaundiced and distended abdomen with the clinical impression of ascites and bilateral ankle edema. Laboratory findings upon admission were as follows: sodium 130 micromole/L (normal range 135-145), abnormal liver enzymes, ALT 125 $\mathrm{U} / \mathrm{L}(0-40)$, aspartate aminotransferase (AST) $526 \mathrm{U} / \mathrm{L}$ (035), alkaline phosphatase (ALK-P) 603 (40-130 U/L), total bilirubin 220 micromole/L (0-17), albumin level of $25 \mathrm{Gr} / \mathrm{L}$ (35-50 Gr/L), high white blood cell count of $15 \times 10^{9} / \mathrm{L}$ (4$\left.10 \times 10^{9} / \mathrm{L}\right)$, coagulation panel and kidney function were normal, hemoglobin $13.6 \mathrm{Gr} \%$ (12-16), a total body a tri-phasic $\mathrm{CT}$ of the liver was completed, which demonstrated a lobu- lated relatively small liver with both hypo and hyperdense lesion highly indicative of pseudo-cirrhosis without signs of vascular insufficiency. Further evaluation for liver decompensation performed during her admission included: negative hepatitis B surface Ag, hepatitis B core antibody, hepatitis $\mathrm{C}$ antibody, cytomegalovirus (CMV) and epstein bar virus (EBV) and negative autoimmune profile (antinuclear, antimitochondrial and anti-parietal antibodies). During her hospitalization, the patient was treated with furosemide diuretics $40 \mathrm{mg}$ once daily, spironolactone $25 \mathrm{mg}$ once daily, lactulose of $30 \mathrm{cc}$ twice daily and intravenous albumin, in addition to several peritoneocentesis, without evidence of spontaneous peritoneal peritonitis and with an elevated serum albumin ascites gradient of $2.1 \mathrm{~g} / \mathrm{dl}$ consistent with portal hypertension as a manifestation of liver decompensation secondary to pseudo-cirrhosis mainly and not from hepatic metastasis per se. Later in her hospitalization, after treatment of hepatic decompensation symptoms, therapy with $5 \mathrm{mg}$ of everolimus was started according to an oncological consultation for the metastatic breast cancer treatment with the plan of perform a schedule PET-CT every six months, and the patient was discharged home in a stable clinical condition. Two month following her discharge the patient died secondary to severe hepatic decompensation and septic shock.

\section{Discussion}

Cirrhosis is considered to be the final stage of any chronic liver disease, characterized by the replacement of the normal hepatic tissue by extensive fibrosis, regenerative nodules formation, and bridging fibrosis leading to distortion of the normal hepatic architecture. ${ }^{[9]}$ The radiological findings of cirrhosis include diffuse nodularity, and a lobulated and relatively small liver. Moreover, signs of portal hypertension, such as splenomegaly, ascites and portosystemic shunts ${ }^{[7]}$ may be seen. Pseudo-cirrhosis is a condition in which there are areas of retracted atrophic liver tissue and scarring in patients with metastatic cancer involving the liver following chemotherapy. This is referred to as "pseudo-cirrhosis" because radiographically it resembles macro nodular cirrhosis and can be associated with hepatic decompensation while lacking of the classic pathologic attributes of cirrhosis $;{ }^{[3]}$ it was most often reported in breast cancer with liver metastasis who were treated with chemotherapeutic agents. However, it was also reported with liver metastasis without chemotherapeutic treatment and with other malignancies associated with liver metastasis, including esophageal, pancreatic, thyroid and small-cell lung cancers. ${ }^{[7,10-12]}$ Both the extent of liver metastasis and the degree of change in hepatic architecture are the single most important predictors which directly correlates with the development of liver 
pseudo-cirrhosis. ${ }^{[5]}$ However, no other associated co-morbid diseases were reported in the literature which predispose to pseudo-cirrhosis. A previous study reported that approximately $75 \%$ of patients with liver metastasis from breast cancer have shown varying degrees of abnormal liver surface, as demonstrated by imaging studies, and that almost 9\% had signs of portal hypertension. ${ }^{[13]}$ Pseudo-cirrhosis has been reported with various chemotherapeutic agents, including cyclophosphamide, adriamycin, 5-fluorouracil, cisplatin, tamoxifen, methotrexate, carmustine, paclitaxel, vinblastine, etoposide, navelbine, and vincristine and ifosfamide..$^{[3,13-18]}$ Still, the exact prevalence is not known, ${ }^{[13]}$ although it may be due to the under-recognition of this occurrence. The precise pathophysiology of pseudo-cirrhosis has not yet been fully elucidated, but previous reports have addressed the patho-mechanisms involved in its development.

Two main mechanisms have been described. The first seems to be secondary to hepatotoxicity from the systemic effects of chemotherapy (i.e., drug-induced liver injury), which causes the development of nodular regenerative hyperplasia in response to chemotherapy-induced hepatic injury; this is thought to be attributed to ischemic atrophy with secondary nodular hyperplasia in regions with favorable blood flow. Nodular regenerative hyperplasia is characterized by the widespread transformation of normal liver parenchyma into hyperplastic regenerative nodules without bridging fibrosis, a feature that distinguishes it from liver cirrhosis. ${ }^{[18,19]}$ Bissonnete et al. in 2012 studied the results from the measurement of the pressure gradients between the hepatic and portal veins for nodular regenerative hyperplasia patients with symptomatic portal hypertension, and they suggested that the mechanism of portal hypertension was associated with portal venopathy and compression of sinusoids by the regenerative nodules. ${ }^{[20]}$

The second patho-mechanism is related to the tumorous hepatic tissue response to the chemotherapeutic agents. ${ }^{[5]}$ Sonnenblick et al. ${ }^{[4]}$ reported the development of pseudocirrhosis in patients with breast cancer and hepatic metastasis in which the supposed mechanism was attributed to tumor shrinkage and subsequent scar formation around the liver lesion which compressed of the adjacent normal liver tissue resulting in atrophy and loss of function. ${ }^{[4]}$ Although cirrhotic changes might appear with both regression and progression of liver metastasis, ${ }^{[13]}$ in order to determine the specific cause of pseudo-cirrhosis, it is essential to perform a liver biopsy for the histological evaluation of the hepatic macro-nodules to assess whether the cirrhosis is due to regression or pro- gression of the liver metastasis. However, since this is an invasive procedure, it is possible that PET-CT will have a role in differentiating the cause of pseudo-cirrhosis, according to a previous report on pseudo-cirrhosis by Sass et al. ${ }^{[5]} \mathrm{In}$ their case, PET-CT scans showed an inhomogeneous uptake consistent with cirrhosis, but no focal areas of increased uptake suggestive of FDG-associated malignancy. Therefore, PET-CT may be helpful in assessing and determining the treatment in such patients, and further studies of the role of PET-CT scan in evaluation of the response to chemotherapy in patients with pseudo-cirrhosis are needed.

While the appearance of clinical features of true cirrhosis is rare, ${ }^{[7]}$ there are several case reports describing the progression of pseudo-cirrhosis to true clinical cirrhosis with portal hypertension manifested as esophageal bleeding and hepatic encephalopathy, suggestive of the fatal life-threatening consequences of pseudo-cirrhosis-irrespective of the pathophysiology. ${ }^{[13,21]}$ Our patient had signs of pseudo-cirrhosis that developed during the period of chemotherapeutic administration. The normal liver contour on PET-CT prior to chemotherapeutic therapy initiation and the absence of uptake on PET-CT following chemotherapeutic treatment suggestive of malignancy indicating that our patient developed chemotherapy induced pseudo-cirrhosis rather than progression of metastasis. Although the main limitation in this case is the absence of a liver biopsy to precisely determine the cause of pseudo-cirrhosis.

This case report addresses the importance of recognizing this entity, not just when breast cancer with liver metastasis is encountered, but with any other malignancies associated liver metastasis, clinicians should be aware of pseudo-cirrhosis. Due to the likelihood of its becoming true clinical cirrhosis as in our case, patients with liver metastasis from chemotherapy should be closely monitored for the development and progression of hepatic failure. Furthermore, the careful choice of chemotherapeutic agents with consideration of hepatic dysfunction is also required.

In conclusion, pseudo-cirrhosis following chemotherapy for hepatic metastasis associated with breast cancer is a rare but significant complication. Early recognition and discontinuation of the chemotherapeutic regimen with supportive hepatic treatment can prevent the life-threatening complication of true cirrhosis-despite the name "pseudo-cirrhosis"-and even lead to full hepatic recovery. Further prospective studies are required to estimate the true impact on patient survival and the need for scoring system that predicts the risk for development of pseudo-cirrhosis. 


\section{REFERENCES}

[1] Jeong WK, Choi SY, Kim J. Pseudocirrhosis as a complication after chemotherapy for hepatic metastasis from breast cancer. Clin Mol Hepatol. 2013; 19(2): 190-194. http://dx.doi.org/10.3350/c $\mathrm{mh} .2013 .19 \cdot 2.190$

[2] Blacher A, Federle MP, Brancatelli G. Hepatic capsular retraction: spectrum of benign and malignant etiologies. Abdom Imaging. 2002; 27(6): 690-699. PMid:12395258 http://dx.doi.org/10.1007 /s00261-001-0094-8

[3] Young ST, Paulson EK, Washington K, et al. CT of the liver in patients with metastatic breast carcinoma treated by chemotherapy: findings simulating cirrhosis. AJR Am J Roentgenol. 1994; 163(6): 13851388. PMid:7992734 http://dx.doi.org/10.2214/ajr.163.6 .7992734

[4] Sonnenblick A, Appelbaum L, Peretz T. Nodular failure on the background of pseudocirrhosis in patients with liver metastasis from breast cancer, who respond to treatment. Onkologie. 2011; 34(4): 199-201. PMid:21447980 http://dx.doi.org/10.1159/000327010

[5] Sass DA, Clark K, Grzybicki D, et al. Diffuse desmoplastic metastatic breast cancer simulating cirrhosis with severe portal hypertension: a case report of pseudocirrohosis. Dig Dis Sci. 2007; 52(3): 749-752. PMid:17265127 http://dx.doi.org/10.1007/s10620-006-9 332-9

[6] Kang SP, Taddei T, McLennan B, et al. Pseudocirrhosis in a pancreatic cancer patient with liver metastasis: a case report of complete resolution of pseudocirrhosis with an early recongnition and management. World J Gastroenterol. 2008; 14(10): 1622-1624. PMid:18330959 http://dx.doi.org/10.3748/wjg.14.1622

[7] Jha P, Poder L, Wang ZL, et al. Radiologic mimics of cirrhosis. AJR Am J Roentgeol. 2010; 194(4): 993-999. PMid:20308502 http://dx.doi.org/10.2214/AJR.09.3409

[8] Fennessy FM, Mortele KJ, Kluckert T, et al. Hepatic capsular retraction in metastatic carcinoma of the breast occurring with increase or decrease in size of subject metastasis. AJR Am J Roentgeol. 2004; 182(3): 651-655. PMid:14975965 http://dx.doi .org/10. 2214 /ajr.182.3.1820651

[9] Ma C, Brunt EM. Histopathologic evaluation of liver biopsy for cirrhosis. Adv Anat Pathol. 2012; 19(4): 220-230. PMid:22692285 http://dx.doi.org/10.1097/PAP.0b013e31825c6bab

[10] Kobashigawa C. Pseudocirrhosis in metastatic esophageal cancer. South Med J. 2010; 103(5): 488-489. PMid:20375937 http://dx .doi.org/10.1097/SMJ .0b013e3181d82d50

[11] Harry BL, Smith ML, Burton Jr JB, et al. Medullary thyroid cancer and pseudocirrhosis: case report and literature review. Curr Oncol.
2012; 19(1): e36-41. PMid:22328846 http://dx.doi .org/10.37 $47 /$ co. 19.840

[12] Liu CH, Chao TY. Education and imaging. Hepatobiliary and pancreatic: Pseudocirrhosis after chemotherapy. J Gastroenterol Hepatol 2011; 26(4): 788. PMid:21418309 http://dx.doi.org/10.1111 /j.1440-1746.2011.06712.x

[13] Qayyum A, Lee GK, Yeh BM, et al. Frequency of hepatic contour abnormalities and signs of portal hypertension at CT in patients receiving chemotherapy for breast cancer metastatic to the liver. Clin Imaging. 2007; 31(1): 6-10. PMid:17189839 http: //dx.doi.org/10.1016/j.clinimag.2006.09.028

[14] Schreiner SA, Gorman B, Stephens DH. Chemotherapy-related hepatotoxicity causing imaging findings resembling cirrhosis. Mayo Clin Proc. 1998; 73(8): 780-783. PMid:9703307 http://dx.doi.org $/ 10.4065 / 73.8 .780$

[15] Washington K. Nodular regenerative hyperplasia in partial hepatectomy specimens. Am J Surg Pathol. 1993; 17(11): 11511158. PMid:8214260 http://dx.doi.org/10.1097/0000047 8-199311000-00008

[16] Rubbia-Brandt L, Audard V, Sartoretti P, et al. Severe hepatic sinusoidal obstruction associated with oxaliplatin-based chemotherapy in patients with metastatic colorectal cancer. Ann Oncol. 2004; 15(3): 460-466. PMid:14998849 http://dx.doi.org/10.1093/annon c/mdh095

[17] Hubert C, Sempoux H, Horsmans Y, et al. Nodular regenerative hyperplasia: a deleterious consequence of chemotherapy for colorectal liver metastases? Liver Int. 2007; 27(7): 938-943. PMid:17696932 http://dx.doi.org/10.1111/j.1478-3231.2007.01511.x

[18] Rosen AA. Nodular regenerative hyperplasia: a cause of ascites and hepatomegaly after chemotherapy for leukemia. Am J Gastroenterol 1991; 86(1): 86-88. PMid:1986559

[19] Key NS, Kelly PM, Emerson PM, et al. Oesophageal varices associated with busulphan-thioguanine combination therapy for chronic myeloid leukaemia. Lancet. 1987; (8567): 1050-1052.

[20] Bissonnette J, Généreux A, Côté J, et al. Hepatic hemodynamics in 24 patients with nodular regenerative hyperplasia and symptomatic portal hypertension. J Gastroenterol Hepatol. 2012; 27(8): 13361340. PMid:22554152 http://dx.doi.org/10.1111/j.1440-1 $746.2012 .07168 . \mathrm{x}$

[21] Chandrakar V, Isaacs C. Breast cancer-related pseudocirrhosis and esophageal varices. Breast. 2005; 11(4): 301-302. PMid:15982406 http://dx.doi.org/10.1111/j.1075-122x.2005.21699.x 